\title{
SISTEMA DE CREENCIAS SOBRE LAS MATEMÁTICAS EN LOS ESTUDIANTES DE EDUCACIÓN BÁSICA
}

\author{
BELIEF SYSTEMS ABOUT MATHEMATICS IN SECONDARY SCHOOL STUDENTS
}

\author{
Jhon Darwin Erazo-Hurtado* y Eliecer Aldana-Bermúdez* *
}

\begin{abstract}
RESUMEN
Este artículo presenta una reflexión sobre la influencia que tienen las creencias de los estudiantes de básica secundaria y media, respecto a las matemáticas y su aprendizaje. Se recogen las apreciaciones, trabajo y conclusiones de diferentes investigaciones donde se ha trabajado en el desarrollo del aprendizaje de las matemáticas y la influencia de los imaginarios y las creencias en el mismo. Los resultados de algunas de estas investigaciones conducen a afirmar que los estudiantes de estos niveles perciben las matemáticas como una materia útil dada su importancia en diferentes aspectos de la cotidianidad como transacciones, reparticiones, estadísticas y demás; pero difícil y mecánica, que se aprende mediante la repetición de ejercicios. Dichas creencias son producto de la experiencia vivida en la escuela e influenciada por el contexto social en el que se desenvuelve el estudiante, donde comúnmente, las matemáticas, no son fácilmente aprehensibles.
\end{abstract}

Palabras clave: Educación matemática, aprendizaje, enseñanza, sistema de creencias, básica secundaria, aprendizaje.

\begin{abstract}
This article describes the influence of the beliefs of elementary and middle school students about mathematics and its learning process. There are collected appreciations, works and findings of several investigations of the development of mathematics learning and the influence of the imaginary and the belief in it. The results of some of these investigations lead to say that these students perceive mathematics as a useful but difficult subject, mechanics that is learned through repetition of exercises. Such beliefs are the product of the experience in school and influenced by the social context in which the student are involved of.
\end{abstract}

Keywords: Imaginary, mathematics education, system belief, secondary learning.

Fecha de recepción: Agosto 05 de 2015 / Fecha de aceptación: Septiembre 18 de 2015

Tipología: Artículo de Reflexión

Para citar el artículo: Erazo, H. J., \& Aldana, B. E. (2015). Sistema de creencias sobre las matemáticas en los estudiantes de educación básica. Praxis. Vol. 11, $163-169$

*Mg. en Ciencias de la educación Universidad del Quindío. Colombia. Email: jderazo@uniquindio.edu.co.

**Dr. En Educación Matemática. Universidad del Quindío. Colombia. Email: eliecerab@uniquindio.edu.co. 


\section{INTRODUCCIÓN}

En diferentes contextos sociales y educativos como las transacciones, el ámbito laboral y la escuela, se escuchan los constantes reclamos acerca de la enseñanza de las matemáticas y la dificultad de los temas en cada uno de los niveles en la educación básica, como lo señala Ayuso, M. (2015), en la escuela, los niños aprenden de memoria las tablas de multiplicar, pero eso no significa que aprendan a multiplicar, pues la mayoría no tiene ni idea de qué está haciendo, memorizan y así no se aprende nada útil. Los ingresos a distintos programas universitarios hacen evidentes las falencias que los estudiantes presentan en torno a los conocimientos en matemáticas. Estos culpan a la mala enseñanza en la escuela media, los profesores al poco interés y estudio por parte de los alumnos y la sociedad al sistema educativo (Gabrielli, P. 2014). Es frecuente que una parte considerable de los estudiantes de un plantel educativo no comprendan el sentido de las actividades propuestas, se desmotiven y esto se refleja en los resultados académicos. En este sentido Díaz, E (2012), realizó un trabajo de investigación en el cual afirma que en las escuelas de nivel básico, principalmente en nivel de secundaria, en su mayoría son las matemáticas las que mayor dificultad de aprendizaje y comprensión presentan para los estudiantes, y como producto de ello se han obtenido índices de aprovechamiento bajos y un alto índice de reprobación, lo que genera luego desmotivación y deserción (Csillang, 2009).

El pánico a la asignatura se debe a que estas tienden a ser difíciles, ya que los estudiantes necesitan ir acumulando una serie de nociones, conceptos y procedimientos, en los cuales tiene que apoyarse para construir nuevos conocimientos (Vergnaud, G. 1990); estos procesos se presentan de forma rápida, por lo que los estudiantes se quedan atrás con frecuencia.

\section{Sentimientos de los estudiantes hacia las matemáticas}

Cuando se habla de creencia se hace referencia a un estado mental del individuo provisto de un contenido idealizado, que representan lo que existe más allá de lo que percibe directamente y, por tanto, susceptible de ser verdadero o falso (Defez, A. 2005). Así, una creencia no puede presentarse independiente de otras, por ende, se suele hablar más de sistemas que de creencias simplemente.

Todo este cúmulo de suposiciones influenciadas hacia las matemáticas por el contexto social del estudiante genera, como mencionan Gil \& Blanco (2006), "sentimientos de intranquilidad, miedo, ansiedad, inseguridad, desconcierto e incertidumbre", que reposan en el profesor que conduce la materia. Lo anterior resalta el hecho de que la naturaleza de esas emociones se da más por los contenidos del área que por los docentes responsables de enseñarlas.

La actitud del estudiante, sus emociones y creencias son factores determinantes para favorecer la comprensión de las matemáticas en cualquier nivel, siendo los primeros años de la educación básica, desde primero hasta quinto, fundamentales para tal fin (Fernández, 2010). De esta forma, Estrada (2002) afirma que la relación entre el dominio afectivo y aprendizaje es cíclica, debido a que los afectos condiciona el comportamiento y la capacidad de aprender, e igualmente el proceso de aprendizaje provoca reacciones afectivas. Estas actitudes y emociones que se dan generalmente en el aula, afectan al estudiante y se van haciendo más sólidas hasta el punto de convertirse en actitudes positivas o negativas hacia las matemáticas, ya en la educación básica secundaria y media. En este sentido, Gómez-Chacón (2006) considera que los comportamientos y las acciones de los estudiantes que participan en las experiencias de aprendizaje organizadas en el aula de clase se ven afectados por las creencias de los docentes, de sus compañeros, padres o representantes y por la de otros actores en su entorno. Además, "para un desarrollo óptimo de la dimensión afectiva son necesarias situaciones que posibiliten el descubrimiento y la liberación de creencias limitativas del estudiante, la incorporación de experiencias vitales así como la estimación de la emoción y el afecto como vehículos del conocimiento matemático" (Gomez-Chacón, 2000). 


\section{Factores y efectos de los sistemas de creencias en los estudiantes en el área de matemáticas}

\section{Actitud}

En cuanto a la actitud, emociones y creencias de los estudiantes, De corte (2004) y Schoenfeld (1992), han destacado aptitudes que el estudiante debe adquirir para tener una buena actitud frente a las matemáticas como son: conocimiento, métodos adecuados de estudio, metacognición, autorregulación y sistema de creencias positivas ante las matemáticas y su aprendizaje. Estos autores señalan que gran parte de la dificultad para aprender y enseñar matemáticas se debe a la falta de una adecuada interconexión que el estudiante debe establecer entre las aptitudes mencionadas.

Los estudiantes con un rendimiento alto y medio adquieren más confianza y gusto por el área, a diferencia de los que tienen un rendimiento bajo, quienes rechazan la materia. En relación con esto, algunos estudios internacionales muestran la existencia de una relación significativa y directa entre las actitudes de los estudiantes y el rendimiento en matemáticas. Por ejemplo, en el estudio del TIMSS (Third International Math and Science Study) realizado en 1995 con la participación de 41 países, se observó una relación positiva entre el gusto por las matemáticas y las puntuaciones obtenidas en las pruebas, así, el puntaje promedio de aquellos estudiantes que manifestaban gusto por dicha materia era superior que el de aquellos que reportaban que las matemática no les gustaba (IEA, 1995).

Sin embargo, Bazán (2001) comenta que “...es preciso considerar que existe la posibilidad de que un alumno pueda alcanzar un nivel de rendimiento satisfactorio y, pese a ello, tener una actitud desfavorable frente a la materia, y viceversa. De esta forma, una actitud positiva no garantiza un mejor rendimiento, aunque sí eleva la probabilidad de que éste se dé”.

\section{Género}

Gómez-Chacón (2006) estima que las niñas manifiestan mayor interés por las matemáticas, pero tienen menos confianza que los niños en obtener buenos resultados. Este punto de vista muestra que, en general, son los hombres quienes obtienen mejores resultados que las mujeres en las pruebas en matemáticas.

Consecuente con lo anterior, el Instituto Colombiano para la Evaluación de la Educación ICFES presentó en 2011 un informe sobre los resultados para el periodo 2005-2010 del examen de estado para la educación media. Allí se detalla los resultados por géneros en el área de matemáticas de las pruebas saber 11 en estos años, como se muestra en la tabla 1. 
Tabla 1

Puntajes promedio y desviaciones estándar en matemáticas por género, calendarios 2005 a 2010

\begin{tabular}{|c|c|c|c|c|c|}
\hline Género & Año & Promedio & $\begin{array}{c}\text { Desviación } \\
\text { Estándar }\end{array}$ & $\begin{array}{l}\text { Promedio } \\
\quad+1 \mathrm{DE}\end{array}$ & Promedio -1 DE \\
\hline \multirow{5}{*}{ Mujeres } & $2005-2$ & 49,6 & 9,5 & 59,1 & 40,1 \\
\hline & 2006-2 & 49,0 & 9,5 & 58,4 & 39,5 \\
\hline & $2007-2$ & 49,5 & 9,6 & 59,1 & 39,9 \\
\hline & $2008-2$ & 49,1 & 9,5 & 58,7 & 39,6 \\
\hline & 2009-2 & 49,4 & 9,5 & 58,9 & 39,9 \\
\hline \multirow{5}{*}{ Hombres } & $2005-2$ & 51,1 & 10,5 & 61,6 & 40,5 \\
\hline & 2006-2 & 52,0 & 10,4 & 62,4 & 41,6 \\
\hline & $2007-2$ & 51,2 & 10,5 & 61,7 & 40,6 \\
\hline & 2008-2 & 51,9 & 10,4 & 62,3 & 41,6 \\
\hline & $2009-2$ & 51,9 & 10,6 & 62,5 & 41,3 \\
\hline \multirow{5}{*}{ Mujeres } & 2006-1 & 49,2 & 9,4 & 58,6 & 39,8 \\
\hline & 2007-1 & 49,8 & 9,6 & 59,3 & 40,2 \\
\hline & 2008-1 & 49,7 & 9,7 & 59,4 & 40,0 \\
\hline & 2009-1 & 49,7 & 9,8 & 59,4 & 30,9 \\
\hline & $2010-1$ & 51,7 & 9,5 & 61,2 & 42,2 \\
\hline \multirow{5}{*}{ Hombres } & 2006-1 & 51,7 & 10,8 & 62,6 & 40,9 \\
\hline & 2007-1 & 51,4 & 10,8 & 62,2 & 40,6 \\
\hline & 2008-1 & 52,0 & 10,5 & 62,6 & 41,5 \\
\hline & $2009-1$ & 52,0 & 10,6 & 62,6 & 41,4 \\
\hline & $2010-1$ & 53,7 & 11,0 & 64,7 & 42,6 \\
\hline
\end{tabular}

Nota. Fuente: ICFES (2011) Examen de estado de la educación media. Resultados del periodo 2005-2010. pp 45.

La anterior tabla muestra la diferencia entre géneros en cuanto a los resultados en las pruebas saber en los periodos 2005-2010 en los primeros semestres, con una diferencia promedio de 2,14 puntos; sin embargo la desviación estándar en las mujeres es menor, lo que indica que su rendimiento es más parejo que el de los hombres. Es de destacar que en el primer periodo de 2010 las mujeres sobrepasan el umbral de los 50 puntos, sin embargo la diferencia con los hombres para este mismo periodo sigue siendo similar, con 2 puntos. En los segundos semestres entre 2005 y 2010, ocurre un fenómeno similar, con una diferencia promedio de 2,3 puntos y la tendencia de la desviación estándar se mantiene. Aunque en este periodo de 5 años, se evidencia una diferencia en los resultados en las pruebas de matemáticas por géneros, también es claro que el desempeño de ambos tiende a aumentar a futuro.

\section{Tipo de colegio}

Otra creencia tiene que ver con la educación en las instituciones no oficiales, donde los estudiantes que asisten a colegios privados adquieren mayor 
facilidad para desempeñarse en matemáticas debido a las facilidades tecnológicas, tiempo extra y acompañamiento que se le da durante su escolaridad. Al respecto, Nuñez et al. (2002) comenta lo siguiente:

...Después de controlar por las características de los individuos, del hogar, de la infraestructura del colegio y el nivel educativo de los docentes, tanto a nivel nacional como a nivel urbano y rural se encuentra una diferencia significativa en favor de los privados. Ésta es atribuible a características no observables, entre las que figuran el sistema de incentivos y ascensos de los maestros. Las diferencias a favor de los colegios privados son más marcadas en los niveles de ingresos medios y altos $\mathrm{y}$, de hecho, en el nivel de ingreso más bajo los estudiantes de colegios públicos obtienen logros superiores.

Los colegios privados son una empresa privada de educación financiada exclusivamente por los padres de los estudiantes. Tienen completa libertad de gestión y cierta libertad de currículum, lo que hace que su mayor preocupación sea estar en los primeros lugares de las pruebas nacionales, con el fin de mantener su estatus y atraer así más estudiantes y evitar la deserción. (Mejores Colegios, 2012). Esto afecta el desarrollo de los cursos de matemáticas en dichos colegios, debido a que estos buscarán mejores resultados en las pruebas nacionales.

\section{Creencias de los estudiantes}

Lo anterior hace referencia a las creencias sociales sobre las matemáticas y el desempeño de niños y niñas en la educación básica secundaria y media; en cuanto a lo que creen los propios estudiantes, Schoenfeld (1992) propone una lista de creencias típicas acerca de la naturaleza de las matemáticas:

- Los problemas matemáticos tienen una única solución correcta

- Existe solo una manera correcta para resolver cualquier problema y es la que el profesor ha mostrado en clase

- Los estudiantes "normales" no pueden esperar entender matemáticas, solo esperan memorizarla y aplicarla mecánicamente y sin entender

- Las matemáticas son una actividad individual, realizada por individuos solitarios

- Los estudiantes que han entendido matemáticas podrán resolver cualquier problema en 5 minutos o menos

- Las matemáticas aprendidas en la escuela tienen poco que ver con el mundo real

- Las pruebas formales no son útiles en el proceso de descubrimiento o invención

Con respecto a la lista anterior, existe una gran influencia sobre cómo se ven los estudiantes a sí mismos como aprendices y su conducta, además, estas creencias dificultan la empatía y por ende el aprendizaje de las matemáticas.

No todos aprenden matemáticas de la misma manera ni a la misma velocidad (Educación inclusiva, 2005). De hecho, Gardner (1993) propone 7 tipos de inteligencia. Sin embargo, cada persona puede desarrollar de modo predominante alguna de ellas y llegar a la superposición sobre las otras. El autor antes mencionado propone el modelo en donde todos los seres humanos están capacitados para el amplio desarrollo de su inteligencia, apoyados en sus capacidades y su motivación, quienes tienen la inteligencia lógica y espacial, serán exitosos en los cursos y carreras que involucran a las matemáticas, ya que estas requieren cierta abstracción, jerarquización, inducción, rigor y perspicacia por parte del estudiante, además, debe tener en cuenta que el aprendizaje matemático es secuencial y acumulativo y esto afecta el desempeño de básica primaria a secundaria y media y luego de este a la educación superior.

Es posible que estas apreciaciones tengan mucho que ver con lo que Lampert (1992) indica:

“...estas creencias están condicionadas por la experiencia escolar. Esto constituye un elemento importante a considerar dentro de una posible estrategia que utilice la resolución de problemas como recurso didáctico".

Así, las creencias de los estudiantes sobre la educación de las matemáticas están determinadas 
por su entorno o mejor, por su contexto social, así como por sus necesidades psicológicas individuales, sus deseos, metas, etc. En otras palabras, los sistemas de creencias están constituidos por certidumbres sobre la naturaleza de las matemáticas, sobre sí mismos y sobre el contexto del estudiante.

\section{CONCLUSIÓN}

En los factores y efectos de los sistemas de creencias en los estudiantes en el área de matemáticas queda en evidencia la importancia de tomar en cuenta los diferentes factores que disponen la influencia afectiva en los procesos educativos, entre ellos: creencias, actitudes, emociones y sus relaciones con otros, estos influyen de forma directa con el proceso de aprendizaje de las matemáticas en los estudiantes de la educación básica y media.

En general, los estudiantes poseen creencias, emociones y actitudes frente a las matemáticas y a la forma como tradicionalmente se expone y evalúa, que puede dificultar su aprendizaje, muestra de ello son los apartes de algunos de los trabajos antes mencionados que concuerdan en que los estudiantes ven a las matemáticas útiles pero de difícil comprensión, la cual se aprende únicamente por repetición y donde las creencias, positivas o negativas, son producto de experiencias vividas durante su formación (Sánchez, 1998).

Es de tener en cuenta que para mejorar el desempeño del docente, las creencias de los estudiantes son tan importantes como los contenidos, por ello, es primordial estimularles a la realización de investigaciones en un contexto que desmitifique las matemáticas y procurar mejores conexiones entre conocimiento y métodos adecuados de estudio para fortalecer sistema de creencias positivos ante las matemáticas y su aprendizaje.

\section{REFERENCIAS BIBLIOGRÁFICAS}

Ayuso. M. (2015). Uno de nuestros mejores profesores señala el gran error en la enseñanza de matemáticas. Diario El Confidencial, Recuperado de: http://www. elconfidencial.com/alma-corazon-vida/2015-01-08/ el-gran-error-que-cometemos-al-ensenar-matematicas-y-8-ideas-para-mejorar-su-aprendizaje_599881.

Bazán, J. Espinosa, G. Farro, C (2001) rendimiento y actitudes hacia la matemática en el sistema escolar peruano. Programa MECEP. Documento de trabajo $\mathrm{N}^{\circ}$ 13. pp 55-70.

Csillang, A. (2009) Los estudiantes de habla hispana con bajo rendimiento y deserción escolar: una perspectiva desde la enseñanza de matemáticas y ciencias. Cuatro de cada diez: Juventud de habla hispana y abandono escolar en Toronto. Universidad de Toronto. pp 49-51.

De Corte, E. (2004). Mainstreams and Perspectives in Reseacher on Learning (Mathematics) from Instruction. Applied Psychology: An International Review, 53(2), pp. 279-310.

Defez, A. (2005) ¿Qué es una creencia? Logos. Anales del Seminario de Metafísica, Vol. 38, pàgs: 199-221. Universidad Complutense de Madrid, Madrid.

Díaz, E. (2012) Factores que podrían afectar el aprendizaje matemático. Primer congreso internacional de Educación "Construyendo inéditos viables". Universidad Autónoma de Chihuahua. México.

EDUCACIÓN INCLUSIVA (2005). Estilos de aprendizaje. Documentos desarrollados para capacitación profesional como parte del Plan Nacional de Inclusión Educativa en 65 centros pilotos del país. Gobierno de Panamá.

Estrada, A. (2002). Análisis de las actitudes y conocimientos estadísticos elementales en la formación del profesorado. Universitat Autonoma, Barcelona, España.

Fernández, I. (2010). Matemáticas en educación primaria. Revista digital Eduinnova [en linea]. Recuperado de: http://www.eduinnova.es/ sep2010/09matematica.pdf

Gabrielli, P. (2014). Algunas reflexiones con respecto a la enseñanza de la matemática. Enseñanza de la matemática. Recuperado de: http://didactica-y-matematica.idoneos.com/ reflexiones_en_torno_a_la_matematica/ 
Gardner, H. (1993). Teoría de las inteligencias múltiples. Barcelona, España. Paidós.

Gil, N. \& Blanco, L. (2006). El papel de la afectividad en la resolución de problemas matemáticos. Revista de Educación, 340. pp. 551-569.

Gómez-Chacón (2000) Matemática emocional. Los afectos en el aprendizaje matemático. Madrid: Narcea.

Gómez-Chacón, I. (2006). Creencias de los estudiantes de matemáticas. La influencia del contexto de clase. Enseñanza de las ciencias. 24(3). 310-315.

IEA (1995) Third International Mathematics and Science Study. TIMSS 1995. Recuperado de: http:// www.iea.nl/timss_1995.html.

ICFES (2011). Examen de estado de la educación media. Resultados del periodo 2005-2010. p.45.

Lampert, M. (1992). Handbook for Research on Mathematics. En Shoenfeld, A.: Learning to think mathematically, Teaching and Learning. D.Grows, Ed. New York:Mac Millan.
MEJORES COLEGIOS (2012). La diferencia entre Colegios públicos, Colegios concertados y Colegios privados. Madrid. Recuperado de: http://www. mejorescolegios.es/la-diferencia-entre-colegiospublicos-colegios-concertados-y-colegios-privados/

Nuñez, J. Steiner, R. Cadena, X. Pardo, R. (2002). ¿Cuáles colegios ofrecen mejor educación en Colombia? Archivos de Economía. Documento 193. Universidad de los Andes. Bogotá.

Sanchez, C. Rawson, W. (2000). ¿Hacia unas Nuevas Matemáticas? Teoría de la educación [en línea]. Número 1(4). Universidad de Salamanca. España.

Schoenfeld, A. (1992). A framework for exploring mathematical cognition. Learning to think mathematically. Berkeley. 34-81. New York: MacMillan.

Vergnaud, G. (1990). La teoría de los campos conceptuales. Recherches en Did'actique des Math'ematiques. 10 (3), pp. $133-170$. 